Nina Kuriyama*

\title{
Testing cointegration in quantile regressions with an application to the term structure of interest rates
}

DOI 10.1515/snde-2013-0107

\begin{abstract}
This paper proposes a cumulated sum (CUSUM) test for the null hypothesis of quantile cointegration. A fully modified quantile estimator is adopted for serial correlation and endogeneity corrections. The CUSUM statistic is composed of the partial sums of the residuals from the fully modified quantile regression. Under the null, the test statistic converges to a functional of Brownian motions. In the application to US interest rates of different maturities, evidence in favor of the expectations hypothesis for the term structure is found in the central part of the distributions of the Treasury bill rate and financial commercial paper rate, but in the tails of the constant maturity rate distribution.
\end{abstract}

Keywords: cointegration; fully modified estimator; quantile regression; term structure.

JEL Classification: C22; E43

\section{Introduction}

The cointegration methodology developed by Engle and Granger (1987) has given rise to numerous studies of long run equilibrium relationships among nonstationary economic variables, such as stock price and market fundamentals (Campbell and Shiller 1987, 1988a,b), short- and long-term interest rates (Engle and Granger 1987; Stock and Watson 1988; Hansen 1992), and aggregate consumption and income (Campbell 1987; Engle and Granger 1987). There is a substantial literature on cointegration tests for time series models. Traditional testing procedures include Engle and Granger (1987) who propose a unit root test on the residuals from the cointegrating regression allowing for heteroskedasticity. Phillips and Ouliaris (1990) analyze the nonstandard asymptotic properties of the Engle-Granger tests. In contrast, Johansen $(1988,1991,1995)$ proposes maximum likelihood test statistics that follow multivariate unit root distributions. Among various approaches the residual based Engle-Granger type tests have been popular due to computational convenience. For example, in a bivariate setting, the residuals $\hat{u}_{t}$ are calculated from the ordinary least squares (OLS) regression of $y_{t}$ on $x_{t}$. If the errors (residuals $\hat{u}_{t}$, in practice) follow a stationary process, denoted $I(0)$, then $y_{t}$ and $x_{t}$ are cointegrated. If the error process has a unit root, denoted $I(1)$, then there is no long run equilibrium between $y_{t}$ and $x_{t}$.

In the Engle-Granger test, if the (augmented) Dickey-Fuller test rejects that the residuals contain a unit root, then we reject the null hypothesis of no cointegration against the alternative of cointegration. However, since long run equilibrium relationship is of particular interest to economists, some authors focus on the null of cointegration using residual based procedures (Park, Ouliaris, and Choi 1988; Park 1990; Shin 1994). More recently, Xiao and Phillips (2002) apply the conventional cumulated sum (CUSUM) test for structural change to cointegrating regression residuals and develop a consistent residual based test for the null of cointegration. Particularly, the fully modified approach proposed by Phillips and Hansen (1990) is used for serial correlation and endogeneity corrections. To examine the fluctuation in the equilibrium error from the cointegrating regression, Xiao and Phillips (2002) calculate the cumulative sum of the residuals from the fully modified OLS regression. Under the null

*Corresponding author: Nina Kuriyama, School of Economics, Mingde Main Building, Renmin University of China, 59 Zhongguancun Street, Beijing 100872, China, e-mail: wangyini@ruc.edu.cn 
of cointegration, the residuals are expected to follow a stable process and the CUSUM test statistic converges to a functional of Brownian motions free of nuisance parameters. Under the alternative of no cointegration, the fluctuations in the residuals have larger order of magnitude and the test statistic diverges to infinity asymptotically. This robust test is easy to implement and has good finite sample performance. Furthermore, Xiao (2009) extends the cointegration methodology to quantile regressions. In the quantile cointegrating regression, leads and lags of the integrated regressors are included to account for endogeneity. The cumulative sum of the resulting residuals has the same asymptotic behavior as that from the fully modified regression considered by Xiao and Phillips (2002). However, in the dynamic model, selecting the lengths of leads and lags can be an issue.

Tests based on the CUSUM statistics were originally introduced to investigate whether a regression relationship is stable over time (Brown and Durbin 1968). Brown, Durbin, and Evans (1975) test constancy of the regression coefficients based on the cumulated sum of squared recursive residuals. Ploberger and Kramer (1992) apply the CUSUM test of parameter stability to OLS residuals from a stationary model. Hao and Inder (1996) propose a diagnostic OLS based CUSUM test for structural change in cointegrated regression models. Although used for different purposes, it should be emphasized that the cointegration models studied by Hao and Inder (1996) and Xiao and Phillips (2002) have the same behavior under the null hypotheses, but have different behaviors under the alternatives.

This paper extends the analysis of Xiao and Phillips (2002) to the case of conditional quantiles, since the long run relationship among nonstationary time series may not be uniform. In practice, locations in the distribution other than the mean may matter for cointegration analysis. To examine the equilibrium relationships across different quantiles of the distribution of the response variable, the CUSUM test is employed to test the null hypothesis of quantile cointegration. Similar to the OLS regression, in the quantile regression with I(1) regressors, due to serial correlation between the regression disturbance and the innovation of the integrated regressors and long run endogeneity in the data, the quantile estimator for the cointegrating coefficients is second-order biased and depends on nuisance parameters. In this case, it is difficult to make inference. To solve this problem, this paper uses a Phillips-Hansen type fully modified quantile estimator. The resulting limit distribution is mixed normal so that it provides a standard inference procedure. The CUSUM test statistic is composed of partial sums of the residuals from the fully modified quantile regression. Under the null of quantile cointegration, the test statistic has the same limit distribution as that from Xiao and Phillips (2002).

A great number of empirical papers find that the interest rate process is I(1) and test for cointegration among interest rates of different terms to maturity. The seminal paper of Campbell and Shiller (1987) demonstrates that present value models of the term structure imply cointegration of short- and long-term interest rates. Engle and Granger (1987), Stock and Watson (1988), Boothe (1991), Hansen (1992), Hall, Anderson, and Granger (1992), Mandeno and Giles (1995), and Downing and Oliner (2007) among others also discuss the expectations theory of the term structure of interest rates. A more complete review of work on the expectations hypothesis of the term structure is provided in Iacone (2009). These papers consider various US interest rate series, including different yield series on the federal funds rate, Treasury bill rate, and commercial paper rate. The results are mixed. This paper applies the residual based quantile cointegration test to several sets of US interest rate data. The quantile version of the CUSUM test rejects the expectations hypothesis of the term structure in certain quantiles of the interest rate distributions.

The remainder of this paper is organized as follows: In Section 2, the model is set up. The asymptotic theory for the fully modified quantile estimator and test statistic is developed. In Section 3, the empirical application is discussed. Section 4 concludes the paper. All proofs are provided in the Appendix.

\section{Theory}

\subsection{The model}

Let $\left\{w_{t}\right\}$ be an $m$-vector time series generated by $w_{t}=\Phi d_{t}+w_{t}^{s}$ for $t=1, \ldots, T$, where $\Phi$ is a coefficient, $d_{t}$ is the deterministic trend such that 


$$
d_{t}= \begin{cases}0 & \text { no trend } \\ 1 & \text { constant term } \\ (1, t)^{\prime} & \text { linear trend }\end{cases}
$$

and $w_{t}^{s}$ is the stochastic component such that $w_{t}^{s}=w_{t-1}^{s}+\xi_{t}$ with $w_{0}^{s}=0$ (Xiao and Phillips 2002). We assume that $\xi_{t}$ satisfies a multivariate invariance principle:

$$
T^{-\frac{1}{2}} \sum_{t=1}^{[T r]} \xi_{t} \Rightarrow B_{w}(r)=B M(0, \Omega)
$$

where " $\Rightarrow$ " denotes weak convergence of the associated probability measures in the space $D[0,1]$ under the Skorohod metric, $[\mathrm{Tr}]$ signifies the integer part of $T r$, and $B_{w}(r), 0 \leq r \leq 1$, is a vector of Brownian motions with covariance matrix $\Omega$. In general, the long run covariance matrix is defined as $\Omega=\lim _{T \rightarrow \infty} T^{-1} E\left(\sum_{t=1}^{T} \xi_{t} \sum_{t=1}^{T} \xi_{t}^{\prime}\right)=\Sigma+\Lambda+\Lambda^{\prime}$, where $\Sigma=\lim _{T \rightarrow \infty} T^{-1} E\left(\sum_{t=1}^{T} \xi_{t} \xi_{t}^{\prime}\right)$ and $\Lambda=\lim _{T \rightarrow \infty} T^{-1} E\left(\sum_{i=1}^{T-1} \sum_{t=1}^{T-i} \xi_{t} \xi_{t+i}^{\prime}\right)$. In addition, the one-sided long run covariance is $\Delta=\Sigma+\Lambda$.

Consider $y_{t}$ as a scalar, $x_{t}$ as a $k$-dimensional vector, and $m=k+1$. We have

$$
w_{t}=\left[\begin{array}{l}
y_{t} \\
x_{t}
\end{array}\right], \quad w_{t}^{s}=\left[\begin{array}{l}
y_{t}^{s} \\
x_{t}^{s}
\end{array}\right], \quad \xi_{t}=\left[\begin{array}{l}
\xi_{1 t} \\
\xi_{2 t}
\end{array}\right], \quad B_{w}(r)=\left[\begin{array}{l}
B_{y}(r) \\
B_{x}(r)
\end{array}\right] .
$$

Thus, the long run covariance $\Omega$ is of the form:

$$
\Omega=\left[\begin{array}{ll}
\omega_{y y}^{2} & \Omega_{y x} \\
\Omega_{x y} & \Omega_{x x}
\end{array}\right] .
$$

Assuming that the elements of $x_{t}^{s}$ are not cointegrated, if $u_{t}=y_{t}^{s}-\beta^{\prime} x_{t}^{s}$ is stationary, then $y_{t}^{s}$ and $x_{t}^{s}$ are cointegrated (Engle and Granger 1987; Phillips and Ouliaris 1990). In the linear regression $y_{t}=\alpha^{\prime} d_{t}+\beta^{\prime} x_{t}+u_{t}=\theta^{\prime} z_{t}+u_{t}$, where $\theta=\left(\alpha^{\prime}, \beta^{\prime}\right)^{\prime}$ and $z_{t}=\left(d_{t}^{\prime}, x_{t}^{\prime}\right)^{\prime}$, the residuals are $\hat{u}_{t}=y_{t}-\hat{\theta}^{\prime} z_{t}$. In the conventional linear case, as discussed by Xiao and Phillips (2002), a CUSUM statistic can be constructed based on the fully modified OLS residuals to test cointegration between $y_{t}$ and $x_{t}$.

However, least squares methods are confined to estimating conditional mean functions for linear models. In order to account for the nonlinearity in the long run relationship, the analysis is generalized to the entire conditional distribution of $y_{t}$, which is represented by quantile $\tau$ with $\tau \in(0,1)$. Using the quantile regression method introduced by Koenker and Bassett (1978), any percentage point in the distribution of the response variable $y_{t}$ conditional on observed covariates $x_{t}$ can be estimated. This method provides a comprehensive picture of the conditional distribution (Koenker and Bassett 1978; Koenker and Hallock 2001). Denote $\mathcal{F}_{t}$ as the information set up to time $t$, so $x_{t} \in \mathcal{F}_{t}$. Let $F(\cdot)$ and $F_{t}(\cdot)=\operatorname{Pr}\left(u_{t}<\cdot \mid \mathcal{F}_{t}\right)$ be the unconditional and conditional cumulative distribution functions of $u_{t}$ and define the $\tau$ th unconditional and conditional quantiles of $u_{t}$ by $Q_{u_{t}}(\tau)=F^{-1}(\tau)$ and $Q_{u_{t}}\left(\tau \mid \mathcal{F}_{t}\right)=F_{t}^{-1}(\tau)$, respectively. With the additional assumption that $F_{t}(\cdot)=F(\cdot)$ for all $1 \leq t \leq T$, the conditional quantile of $y_{t}$ is $Q_{y_{t}}\left(\tau \mid \mathcal{F}_{t}\right)=\alpha^{\prime} d_{t}+F^{-1}(\tau)+\beta^{\prime} x_{t}$ for $t=1, \ldots, T$. Under this restrictive specification, all quantiles share the same slope coefficient $\beta$. However, the slope coefficient may depend on the innovation process $u_{t}$. In this case, the conditional quantile of the error term can be modeled as $Q_{u_{t}}\left(\tau \mid \mathcal{F}_{t}\right)=d_{t}^{\prime}(\alpha(\tau)-\alpha)+x_{t}^{\prime}(\beta(\tau)-\beta)$. Then, the conditional quantile function of $y_{t}$ is $Q_{y_{t}}\left(\tau \mid \mathcal{F}_{t}\right)=\alpha^{\prime}(\tau) d_{t}+\beta^{\prime}(\tau) x_{t}$. Define $u_{t}(\tau)=u_{t}-F_{t}^{-1}(\tau)=y_{t}-\alpha^{\prime}(\tau) d_{t}-\beta^{\prime}(\tau) x_{t}$ so that $Q_{u_{t}(\tau)}\left(\tau \mid \mathcal{F}_{t}\right)=0$. Consequently, we have the following quantile regression model:

$$
y_{t}=\alpha^{\prime}(\tau) d_{t}+\beta^{\prime}(\tau) x_{t}+u_{t}(\tau)=\theta^{\prime}(\tau) z_{t}+u_{t}(\tau),
$$

where $\theta(\tau)=\left(\alpha^{\prime}(\tau), \beta^{\prime}(\tau)\right)^{\prime}$. The quantile dependent regression coefficient vector $\theta(\tau)$ can characterize the possibly nonlinear long run relationship between $y_{t}$ and $x_{t}$. Nonetheless, for each $\tau$ the conditional quantile of $y_{t}$

1 The calculations involving the conditional quantile regressions are only valid if the conditional distribution of $u_{t}$ is smooth. Assumption 2 in the next subsection is sufficient for this smoothness. 
is formulated as a linear function of $\theta(\tau)$ so that the unknown coefficients can be estimated by linear programming (Koenker and Hallock 2001).

\subsection{Estimation method}

Consider the following objective function with asymmetric weights on positive and negative residuals:

$$
S_{T}(\tau, \theta)=\sum_{t=1}^{T} \rho_{\tau}\left(y_{t}-z_{t}^{\prime} \theta\right) .
$$

Here, function $\rho_{\tau}(\cdot)$ is the asymmetric absolute deviation loss function defined by $\rho_{\tau}(u)=u(\tau-I(u<0))$, also known as the check function (Koenker and Bassett 1978). Define $\psi_{\tau}(u)=\tau-I(u<0)$. For the $\tau$ th quantile, the unknown regression coefficients are estimated by minimizing the sum of asymmetrically weighted absolute residuals such that

$$
\hat{\theta}(\tau)=\arg \min _{\theta} S_{T}(\tau, \theta)
$$

where $\hat{\theta}(\tau)=\left(\hat{\alpha}^{\prime}(\tau), \hat{\beta}^{\prime}(\tau)\right)^{\prime}$.

\subsection{Assumptions}

The following assumptions are imposed in order to derive the asymptotic distribution of the test statistic.

Assumption 1 Define $\xi_{t}(\tau)=\left(\psi_{\tau}\left(u_{t}(\tau)\right), \xi_{2 t}^{\prime}\right)^{\prime}$. The partial sum process of $\xi_{t}(\tau)$ satisfies a multivariate invariance principle:

$$
T^{-\frac{1}{2}} \sum_{t=1}^{[T r]} \xi_{t}(\tau) \Rightarrow\left[\begin{array}{c}
B_{\psi}(r) \\
B_{x}(r)
\end{array}\right]=B M\left(0, \Omega_{\tau}\right),
$$

where $\left(B_{\psi}(r), B_{x}^{\prime}(r)\right)^{\prime}$ is a vector of Brownian motions with covariance matrix

$$
\Omega_{\tau}=\left[\begin{array}{cc}
\omega_{\psi}^{2} & \Omega_{\psi x} \\
\Omega_{x \psi} & \Omega_{x x}
\end{array}\right],
$$

where $\omega_{\psi}^{2}=\lim _{T \rightarrow \infty} T^{-1} E\left[\sum_{t=1}^{T} \psi_{\tau}\left(u_{t}(\tau)\right) \sum_{t=1}^{T} \psi_{\tau}\left(u_{t}(\tau)\right)\right]$ is the long run variance of $\psi_{\tau}\left(u_{t}(\tau)\right), \Omega_{\psi x}=\Omega_{x \psi}^{\prime}=$ $\lim _{T \rightarrow \infty} T^{-1} E\left[\sum_{t=1}^{T} \psi_{\tau}\left(u_{t}(\tau)\right) \sum_{t=1}^{T} \xi_{2 t}^{\prime}\right]$, and $\Omega_{x x}=\lim _{T \rightarrow \infty} T^{-1} E\left[\sum_{t=1}^{T} \xi_{2 t} \sum_{t=1}^{T} \xi_{2 t}^{\prime}\right]>0$.

Assumption 2 The conditional distribution function, $F_{t}(u)=\operatorname{Pr}\left(u_{t}<u \mid \mathcal{F}_{t}\right)$, is absolutely continuous and has a continuous density function $f_{t}(u)$ such that $0<f_{t}\left(F_{t}^{-1}(\tau)\right)<\infty$ for $t=1, \ldots, T$.

Assumption 3 The density function $f_{t}\left(v_{T}\right)$ is uniformly integrable for any sequence $v_{T} \rightarrow F_{t}^{-1}(\tau)$ and $E\left[f_{t}^{\gamma}\left(F_{t}^{-1}(\tau)\right)\right]<\infty$ for some $\gamma>1$, for $t=1, \ldots, T$.

Assumptions 2 and 3 are two standard technical assumptions in the quantile regression literature such as Xiao (2009), Qu (2008), and Oka and Qu (2011)2. These two assumptions ensure that the conditional density function is uniformly continuous, bounded, and integrable in some neighborhood of the $\tau$ th quantile.

2 Assumption 2 corresponds to Assumption B in Xiao (2009) and Assumption 2 in Oka and Qu (2011). Assumption 3 corresponds to Assumption C in Xiao (2009). Assumptions 1 and 2 in Qu (2008) allow heteroskedasticity and ensure smoothness of the conditional densities. 


\subsection{Theoretical results}

If the above assumptions hold, the estimates of the regression coefficients will converge to their true values. Let $D_{T}$ be a diagonal matrix. The presence of $D_{T}$ is due to the different convergence rates of the coefficients associated with the deterministic trend and integrated regressors. When the regression model contains an intercept such that $d_{t}=1$, we have $D_{T}=\operatorname{diag}\left(T^{\frac{1}{2}}, T I_{k}\right)$. In the case of a linear trend such that $d_{t}=(1, t)^{\prime}$, we have $\operatorname{diag}\left(1, T^{-1}\right) d_{[T r]} \Rightarrow B_{d}(r)=(1, r)^{\prime}$. Since the convergence rate of the coefficient on the trending variable is $T$, the diagonal matrix becomes $D_{T}=\operatorname{diag}\left(T^{\frac{1}{2}}, T I_{k+1}\right)$. From now on the integral " " denotes integration from 0 to 1 and the argument of the Brownian motions are " $r$," $0 \leq r \leq 1$, unless otherwise specified. Similar to Theorem 1 in Xiao (2009), under the null hypothesis of cointegration denoted $H_{0}$, the limit distribution of the coefficient estimator is given by the following representation:

Theorem 1 Under $H_{0}$ and Assumptions 1-3, $\hat{\theta}(\tau)$ is a consistent estimator of $\theta(\tau)$ and

$$
D_{T}(\hat{\theta}(\tau)-\theta(\tau)) \Rightarrow\left[f\left(F^{-1}(\tau)\right) \int B_{z} B_{z}^{\prime}\right]^{-1}\left[\int B_{z} d B_{\psi}+\bar{\Delta}_{x \psi}\right],
$$

where $B_{z}=\left(B_{d}^{\prime}, B_{x}^{\prime}\right)^{\prime}, \bar{\Delta}_{x \psi}=\left(0, \Delta_{x \psi}^{\prime}\right)^{\prime}$, and $\Delta_{x \psi}=\sum_{t=0}^{\infty} E\left(\xi_{2 t} \psi_{\tau}\left(u_{0}(\tau)\right)\right)$ is the one-sided long run covariance between $\xi_{2 t}$ and $\psi_{\tau}\left(u_{t}(\tau)\right)$.

The asymptotic representation is composed of integrals of Brownian motions and a bias term. In particular, the asymptotic distribution of the cointegrating coefficient estimator $\hat{\beta}(\tau)$ is as follows:

$$
T(\hat{\beta}(\tau)-\beta(\tau)) \Rightarrow\left[f\left(F^{-1}(\tau)\right) \int_{\underline{B}_{x d}} \underline{B}_{x d}^{\prime}\right]^{-1}\left[\int \underline{B}_{x d} d B_{\psi}+\Delta_{x \psi}\right],
$$

where $\underline{B}_{x d}=B_{x}-\left(\int B_{x} d^{\prime}\right)\left(\int d B_{d}^{\prime}\right)^{-1} B_{d}$ is a $k$-dimensional demeaned or detrended Brownian motion. In the presence of serial cross correlation, the limit distribution of $\hat{\beta}(\tau)$ has a second-order bias term $\Delta_{x \psi}$. Also, the above limit distribution depends on the nuisance parameter $\Omega$, since the distribution of $\int \underline{B}_{x d} d B_{\psi}$ depends on the correlation between $B_{x}$ and $B_{\psi}$, which is unknown in general. In order to develop useful inference procedures, the bias and nuisance parameter need to be removed. Moreover, to make the model parsimonious, instead of including leads and lags of the nonstationary regressors as in the quantile-varying cointegration model of equation (9) from Section 3.1 of Xiao (2009), this paper exploits the Phillips-Hansen type fully modified estimator to correct the serial correlation and endogeneity. As suggested by Phillips and Hansen (1990) and Phillips and Loretan (1991), the fully modified estimator displays superior properties than those of the usual estimator ${ }^{3}$.

Following Phillips and Hansen (1990), define $\psi_{\tau}^{+}\left(u_{t}(\tau)\right)=\psi_{\tau}\left(u_{t}(\tau)\right)-\Omega_{\psi x} \Omega_{x x}^{-1} \xi_{2 t}$ so that $\psi_{\tau}^{+}\left(u_{t}(\tau)\right)$ is uncorrelated with $\xi_{2 t}$ and has variance $\omega_{\psi \cdot x}^{2}=\omega_{\psi}^{2}-\Omega_{\psi x} \Omega_{x x}^{-1} \Omega_{x \psi}$. Then we have

$$
\begin{aligned}
T^{-1} \sum_{t=1}^{T} x_{t} \psi_{\tau}^{+}\left(u_{t}(\tau)\right) & \Rightarrow \int B_{x} d B_{\psi \cdot x}+\Delta_{x \psi}^{+} \\
& =\omega_{\psi \cdot x} \int B_{x} d W+\Delta_{x \psi}^{+},
\end{aligned}
$$

where $B_{\psi \cdot x}=B_{\psi}-\Omega_{\psi x} \Omega_{x x}^{-1} B_{x}$ is a Brownian motion independent of $B_{x}, W=B_{\psi \cdot x} / \omega_{\psi \cdot x}$ is a standard Brownian motion, and $\Delta_{x \psi}^{+}=\Delta_{x \psi}-\Omega_{\psi x} \Omega_{x x}^{-1} \Delta_{x x}$ is the one-sided long run covariance between $\xi_{2 t}$ and $\psi_{\tau}^{+}\left(u_{t}(\tau)\right)$.

The long run variance $\omega_{\psi}^{2}$ can be estimated by the following kernel estimator:

$$
\hat{\omega}_{\psi}^{2}=\sum_{h=-M}^{M} K\left(\frac{h}{M}\right) \hat{\Gamma}_{\psi \psi}(h),
$$

3 Phillips and Loretan (1991) show the advantage of the fully modified OLS estimator over the usual OLS estimator by Monte Carlo simulations. In the quantile regression case, the finite sample bias and root mean square error of the fully modified quantile estimator are significantly smaller than those of the usual conditional quantile estimator (Kuriyama 2015). 
where $\hat{\Gamma}_{\psi \psi}(h)=T^{-1} \sum_{t=1}^{T-h} \psi_{\tau}^{2}\left(\hat{u}_{t+h}(\tau)\right)$ with quantile regression residual $\hat{u}_{t}(\tau)=y_{t}-\hat{\theta}^{\prime}(\tau) z_{t}, K(\cdot)$ is a kernel function defined on $[-1,1]$ with $K(0)=1$, and $M$ is the bandwidth that $M \rightarrow \infty$ and $M / T \rightarrow 0$. The other elements in the long run variance $\Omega_{\tau}$ and the one-sided long run covariance can be estimated in the similar fashion. Let $\hat{\Delta}_{x \psi}^{+}=\hat{\Delta}_{x \psi}-\hat{\Omega}_{\psi x} \hat{\Omega}_{x x}^{-1} \hat{\Delta}_{x x}$, where $\hat{\Omega}_{\psi x}=\hat{\Omega}_{x \psi}^{\prime}, \hat{\Omega}_{x x}, \hat{\Delta}_{x \psi}$, and $\hat{\Delta}_{x x}$ are the kernel estimators:

$$
\begin{aligned}
& \hat{\Omega}_{x \psi}=\sum_{h=-M}^{M} K\left(\frac{h}{M}\right) \hat{\Gamma}_{x \psi}(h), \hat{\Delta}_{x \psi}=\sum_{h=0}^{M} K\left(\frac{h}{M}\right) \hat{\Gamma}_{x \psi}(h), \\
& \hat{\Omega}_{x x}=\sum_{h=-M}^{M} K\left(\frac{h}{M}\right) \hat{\Gamma}_{x x}(h), \hat{\Delta}_{x x}=\sum_{h=0}^{M} K\left(\frac{h}{M}\right) \hat{\Gamma}_{x x}(h),
\end{aligned}
$$

where $\hat{\Gamma}_{x \psi}(h)=T^{-1} \sum_{t=1}^{T-h} \Delta x_{t} \psi_{\tau}\left(\hat{u}_{t+h}(\tau)\right)$ and $\hat{\Gamma}_{x x}(h)=T^{-1} \sum_{t=1}^{T-h} \Delta x_{t} \Delta x_{t+h}^{\prime}$. Under the null of cointegration $\hat{\omega}_{\psi}^{2}, \hat{\Omega}_{x \psi}, \hat{\Delta}_{x \psi}, \hat{\Omega}_{x x}$, and $\hat{\Delta}_{x x}$ are consistent estimators of $\omega_{\psi}^{2}, \Omega_{x \psi}, \Delta_{x \psi}, \Omega_{x x}$, and $\Delta_{x x}^{4}$. As suggested by Andrews (1991) and Xiao and Phillips (2002), the Bartlett kernel and a plug-in bandwidth are adopted such that

and $M=O\left(T^{\frac{1}{3}}\right)$.

$$
K(u)= \begin{cases}1-|u| & \text { for }|u| \leq 1 \\ 0 & \text { otherwise }\end{cases}
$$

The regression coefficient estimator after the modification, $\hat{\theta}^{+}(\tau)=\left(\hat{\alpha}^{\prime}(\tau), \hat{\beta}^{\prime+}(\tau)\right)^{\prime}$, is

$$
\hat{\theta}^{+}(\tau)=\hat{\theta}(\tau)-\left[\overline{f\left(F^{-1}(\tau)\right)} \sum_{t=1}^{T} z_{t} z_{t}^{\prime}\right]^{-1}\left[\sum_{t=1}^{T} z_{t} \hat{\Omega}_{\psi x} \hat{\Omega}_{x x}^{-1} \Delta x_{t}+T \overline{\hat{\Delta}}_{x \psi}^{+}\right],
$$

where $\overline{\hat{\Delta}}_{x \psi}^{+}=\left(0, \hat{\Delta}_{x \psi}^{\prime+}\right)^{\prime}$. In particular, the fully modified estimator of the coefficients associated with the I(1) regressors is given by

$$
\hat{\beta}^{+}(\tau)=\hat{\beta}(\tau)-\left[\overline{f\left(F^{-1}(\tau)\right)} \sum_{t=1}^{T} \underline{x}_{t}^{d} \underline{x}_{t}^{\prime d}\right]^{-1}\left[\sum_{t=1}^{T} \underline{x}_{t}^{d} \hat{\Omega}_{\psi x} \hat{\Omega}_{x x}^{-1} \Delta x_{t}+T \hat{\Delta}_{x \psi}^{+}\right],
$$

where $\underline{x}_{t}^{d}$ denotes the demeaned or detrended regressors and $\overline{f\left(F^{-1}(\tau)\right)}$ is a nonparametric consistent estimator of the density function $f\left(F^{-1}(\tau)\right)$. The density function can be estimated using the Gaussian kernel and Silverman's "rule-of-thumb" bandwidth.

Consequently, the fully modified estimator $\hat{\beta}^{+}(\tau)$ follows a mixed normal distribution in the limit such that

$$
\begin{aligned}
T\left(\hat{\beta}^{+}(\tau)-\beta(\tau)\right) & \Rightarrow\left[f\left(F^{-1}(\tau)\right) \int \underline{B}_{x d} \underline{B}_{x d}^{\prime}\right]^{-1} \omega_{\psi \cdot x} \underline{B}_{x d} d W \\
& \sim M N\left(0, \frac{\omega_{\psi \cdot x}^{2}}{f\left(F^{-1}(\tau)\right)^{2}}\left[\int \underline{B}_{x d} \underline{B}_{x d}^{\prime}\right]^{-1}\right),
\end{aligned}
$$

where $\omega_{\psi \cdot x}^{2}$ is estimated by $\hat{\omega}_{\psi \cdot x}^{2}=\hat{\omega}_{\psi}^{2}-\hat{\Omega}_{\psi x} \hat{\Omega}_{x x}^{-1} \hat{\Omega}_{x \psi}$, which is calculated by the nonparametric kernel method. Therefore, the asymptotic distribution of $\hat{\theta}^{+}(\tau)$ is as follows:

Theorem 2 Under $H_{0}$ and Assumptions $1-3, \hat{\theta}^{+}(\tau)$ is a consistent estimator of $\theta(\tau)$ and

$$
\begin{aligned}
D_{T}\left(\hat{\theta}^{+}(\tau)-\theta(\tau)\right) & \Rightarrow\left[f\left(F^{-1}(\tau)\right) \int B_{z} B_{z}^{\prime}\right]^{-1} \omega_{\psi \cdot x} \int B_{z} d W \\
& \sim M N\left(0, \frac{\omega_{\psi \cdot x}^{2}}{f\left(F^{-1}(\tau)\right)^{2}}\left[\int B_{z} B_{z}^{\prime}\right]^{-1}\right),
\end{aligned}
$$

where $B_{z}=\left(B_{d}^{\prime}, B_{x}^{\prime}\right)^{\prime}$ and $\omega_{\psi \cdot x}^{2}=\omega_{\psi}^{2}-\Omega_{\psi x} \Omega_{x x}^{-1} \Omega_{x \psi}$.

4 Andrews (1991) discusses consistent covariance matrix estimation using similar kernel estimators in more general cases of heteroskedasticity and autocorrelation of unknown form. 
In the linear regression case, the cumulated sum of the residuals, which is $T^{-\frac{1}{2}} \sum_{t=1}^{n} \hat{u}_{t}, n=1, \ldots, T$, converges under the null of cointegration and diverges to infinity under the alternative. Analogously, in the quantile regression model, the cumulated sum of $\psi_{\tau}\left(\hat{u}_{t}(\tau)\right)$ satisfies the following conditions:

$$
\max _{n=1, \ldots, T} T^{-\frac{1}{2}}\left|\sum_{t=1}^{n} \psi_{\tau}\left(\hat{u}_{t}(\tau)\right)\right|=\left\{\begin{array}{ll}
O_{p}(1) & \text { under } H_{0} \\
O_{p}(T) & \text { under } H_{1}
\end{array} .\right.
$$

Under $H_{0}$, the cumulated sum of the fully modified residuals converges to a functional of Brownian motions. For the quantile regression model, the residuals from the fully modified quantile regression is calculated as $\hat{u}_{t}^{+}(\tau)=y_{t}^{+}-z_{t}^{\hat{\theta}^{+}}(\tau)$, where $y_{t}^{+}=y_{t}-\hat{\Omega}_{\psi x} \hat{\Omega}_{x x}^{-1} \Delta x_{t}$. The CUSUM test statistic is given by

$$
C S_{T}(\tau)=\max _{n=1, \ldots, T} \frac{1}{\hat{\omega}_{\psi \cdot x} \sqrt{T}}\left|\sum_{t=1}^{n} \psi_{\tau}\left(\hat{u}_{t}^{+}(\tau)\right)\right| .
$$

In particular,

$$
\begin{aligned}
T^{-\frac{1}{2}} \sum_{t=1}^{[T r]} \psi_{\tau}\left(\hat{u}_{t}^{+}(\tau)\right) & \Rightarrow B_{\psi \cdot x}-\left[\int d B_{\psi \cdot x} B_{z}^{\prime}\right]\left[\int B_{z} B_{z}^{\prime}\right]^{-1} \int_{0}^{r} B_{z} \\
& =\omega_{\psi \cdot x}\left\{W_{1}-\left[\int d W_{1} S^{\prime}\right]\left[\int S S^{\prime}\right]^{-1} \int_{0}^{r} S\right\},
\end{aligned}
$$

where $S=\left(B_{d}^{\prime}, W_{2}^{\prime}\right)^{\prime}$ and $W_{1}$ and $W_{2}$ are one and $k$-dimensional standard Brownian motions that are independent of each other.

Define

$$
\underline{W}(r)=W_{1}-\left[\int d W_{1} S^{\prime}\right]\left[\int S S^{\prime}\right]^{-1} \int_{0}^{r} S .
$$

Then, for a certain quantile $\tau$, the asymptotic representation of the CUSUM test statistic is as follows:

Theorem 3 Under $H_{0}$ and Assumptions 1-3,

$$
C S_{T}(\tau) \Rightarrow \sup _{0 \leq r \leq 1}|\underline{W}(r)| .
$$

For each quantile level $\tau$, the asymptotic distribution is the same as that from the linear case. Simulated critical values are tabulated in Tables 1 and 2 from Hao and Inder (1996) and Table 1 from Xiao and Phillips (2002). Hao and Inder (1996) also consider the CUSUM statistic, however, for testing the null hypothesis of parameter constancy. Under the null of no structural change, the test statistic from their model has the same asymptotic distribution as the cumulative sum statistic here. The limit distributions are different under the alternatives. The empirical size and power properties of the CUSUM test for cointegration are evaluated via simulation by Xiao and Phillips (2002). In the case of quantile regression, the finite sample properties of the test are similar to those from the fully modified OLS regression.

Under $H_{1}$, the cumulated sum of the fully modified residuals diverges to infinity. Note that the fully modified residuals of the quantile regression model can be rewritten as:

$$
\hat{u}_{t}^{+}(\tau)=\hat{u}_{t}(\tau)-\hat{\Omega}_{\psi x} \hat{\Omega}_{x x}^{-1} \Delta x_{t}+z_{t}^{\prime}\left[\overline{f\left(F^{-1}(\tau)\right)} \sum_{t=1}^{T} z_{t} z_{t}^{\prime}\right]^{-1}\left[\sum_{t=1}^{T} z_{t} \hat{\Omega}_{\psi x} \hat{\Omega}_{x x}^{-1} \Delta x_{t}+T \overline{\hat{\Delta}}_{x \psi}^{+}\right] .
$$

As shown in Lemma 1 of Xiao and Phillips (2002), under $H_{1}$, as $T \rightarrow \infty, M \rightarrow \infty$, and $M / T \rightarrow 0$,

$$
\hat{\Omega}_{\psi x}=\hat{\Omega}_{x \psi}^{\prime}=O_{p}(M), \quad \hat{\Delta}_{x \psi}=O_{p}(M), \quad \hat{\omega}_{\psi \cdot x}^{2}=O_{p}(T M) .
$$

5 Proof of this result is included in the proofs of Theorem 3 and Theorem 4 in the Appendix. 
Furthermore, we have

$$
\hat{\Omega}_{\psi x} \hat{\Omega}_{x x}^{-1} \chi_{[T r]}=O_{p}\left(T^{\frac{1}{2}} M\right) \text { and } \sum_{t=1}^{[T r]} z_{t}^{\prime}\left[\widehat{f\left(F^{-1}(\tau)\right)} \sum_{t=1}^{T} z_{t} z_{t}^{\prime}\right]^{-1}\left[\sum_{t=1}^{T} z_{t} \hat{\Omega}_{\psi x} \hat{\Omega}_{x x}^{-1} \Delta x_{t}+T \overline{\hat{\Delta}}_{x \psi}^{+}\right]=O_{p}\left(T^{\frac{1}{2}} M\right) .
$$

The following theorem presents consistency of the test:

Theorem 4 Under $H_{1}$ and Assumptions 1-3, as $T \rightarrow \infty, \operatorname{Pr}\left(C S_{T}(\tau)>A_{T}\right) \rightarrow 1$ for any nonstochastic sequence $A_{T}=O\left(T^{\frac{1}{2}} M^{-\frac{1}{2}}\right)$.

Therefore, under the alternative of no cointegration the test statistic diverges and the divergence rate depends on the bandwidth.

\section{Empirical study}

The theoretical model can be applied to examining the term structure of interest rates, and, more specifically, to testing the hypothesis of cointegration of short- and long-term interest rates. The expectations theory of the term structure of interest rates suggests that interest rates should be cointegrated if can be characterized as I(1) processes.

According to the generalized-present-value (GPV) model, any long-term yield can be expressed as a function of current and expected short-term yields (Boothe 1991). Engle and Granger (1987) and Campbell and Shiller (1987) test the model of the term structure and find that the yields of domestic bonds that differ only by term to maturity are intimately linked. Since interest rates in the US are usually characterized as nonstationary processes, the spread between these interest rates of different terms to maturity should be stationary if the expectations theory of the term structure holds. This means that short- and long-term rates are cointegrated.

Many papers have tested the expectations hypothesis for the term structure using US yield series on the federal funds rate (Hansen 1992), Treasury bill rate (Stock and Watson 1988; Boothe 1991; Hall, Anderson, and Granger 1992; Hansen 1992; Mandeno and Giles 1995), and commercial paper rate (Downing and Oliner 2007). In this paper, three sets of data are considered. The data comprises daily observations of interest rates of different yields from the U.S. Department of the Treasury and the Board of Governors of the Federal Reserve System. The interest rate statistics include daily Treasury bill rates, ${ }^{6}$ U.S. government securities/Treasury constant maturities (nominal) interest rates, ${ }^{7}$ and financial commercial paper rates. ${ }^{8}$ The frequency is one

6 Data source is the U.S. Department of the Treasury. US daily 4-, 13-, and 26-week Treasury bill rates are from 01/02/2002 to 04/30/2012. The number of observations is 2585 . Both bank discount and coupon equivalent rates are considered. Secondary market quotations on the most recently auctioned Treasury Bills are obtained at approximately 3:30 PM each business day by the Federal Reserve Bank of New York. "The Bank Discount rate is the rate at which a Bill is quoted in the secondary market and is based on the par value, amount of the discount and a 360-day year. The Coupon Equivalent, also called the Bond Equivalent, or the Investment Yield, is the bill's yield based on the purchase price, discount, and a 365- or 366-day year. The Coupon Equivalent can be used to compare the yield on a discount bill to the yield on a nominal coupon bond that pays semiannual interest." (U.S. Department of the Treasury, http://www.treasury.gov/resource-center/data-chart-center/interest-rates/Pages/TextView. aspx?data=billrates, 07 May 2012.)

7 Data source is the Board of Governors of the Federal Reserve System. US daily 3-, 6-, and 12-month Treasury bill constant maturity rates are from $01 / 04 / 1982$ to $04 / 30 / 2012$. The number of observations is 7584 . The market yields are quoted on investment basis and are interpolated by the Treasury from the daily Treasury yield curve. The yield curve relates the yield on a security to its time to maturity. It is based on the closing market bid yields on actively traded Treasury securities in the over-the-counter market. The market yields are calculated from composites of quotations obtained by the Federal Reserve Bank of New York. The constant maturity Treasury rates, or CMTs, are also referred to as the Treasury yield curve rates. The Treasury will restrict the use of negative input yields for securities used in deriving interest rates for the nominal constant maturity Treasury series. "Any CMT input points with negative yields will be reset to zero percent prior to use as inputs in the CMT derivation.” (U.S. Department of the Treasury, http://www.treasury.gov/resource-center/data-chart-center/interest-rates/Pages/TextView.aspx?data=yield, 07 May 2012.) 8 Data source is the Board of Governors of the Federal Reserve System. US daily 30-, 60-, and 90-day AA financial commercial paper rates are from 01/02/1997 to 04/30/2012. The number of observations is 3748 . 
business day. Unavailable or missing observations of holidays are omitted. The longest series, which is the Treasury bill constant maturity rate, is from January 4, 1982 to April 30, 2012 and contains 7584 observations. The shortest series, which is the Treasury bill rate, is from January 2, 2002 to April 30, 2012 and contains 2585 observations.

The regression model contains an intercept term but no time trend $\left(d_{t}=1\right)$, as a result of which $y_{t}=\alpha(\tau)+\beta(\tau) x_{t}+u_{t}(\tau)$. Nine representative quantile levels, $\tau=0.1,0.2, \ldots, 0.9$, are considered. The bandwidth for the fully modified kernel estimator is $M=2 T^{\frac{1}{3}}$.

As a pretest to investigate the properties of the interest rate series, the augmented Dickey-Fuller (ADF) test does not reject that the logarithm of each rate has a unit root, but strongly rejects a unit root in the first difference of the series. Thus, the interest rates are I(1).

The estimation and test results are summarized in Tables $1-3$. For each quantile $\tau$, the fully modified coefficient estimate $\hat{\beta}^{+}(\tau)$ and the CUSUM test statistic $C S_{T}(\tau)$ are reported. Let * denote the case where the CUSUM test rejects the null of cointegration at $5 \%$ level and let ${ }^{* *}$ denote rejection at $1 \%$ level. For each instrument, the evidence in support of the expectations hypothesis for the term structure is mixed over different quantiles, since the CUSUM test fails to reject cointegration in various positions over the interest rate distributions.

As Tables 1 and 2 show, short- and long-term bank discount and coupon equivalent Treasury bill rates and financial commercial rates are generally cointegrated in the central part of the distribution, but not in the tails. In the first panel of Table 1, 4-, 13-, and 26-week Treasury bill bank discount rates are cointegrated with each other for the inner quanitles. Also, the $90 \%$ quantile of the 13-week bank discount rate is cointegrated with the 4-week rate. Similarly, the $90 \%$ quantile of the 26 -week rate and the 4-week rate are cointegrated.

Table 1: Cointegration among Treasury bill rates (yields: 4, 13, 26 weeks).

\begin{tabular}{|c|c|c|c|c|c|c|c|c|c|c|}
\hline$y-x$ & & $\tau=0.1$ & 0.2 & 0.3 & 0.4 & 0.5 & 0.6 & 0.7 & 0.8 & 0.9 \\
\hline \multicolumn{11}{|c|}{ Bank discount } \\
\hline \multirow[t]{2}{*}{$4-13$} & $\hat{\beta}^{+}(\tau)$ & 0.9277 & 0.9519 & 0.9657 & 0.9743 & 0.9823 & 0.9917 & 1.0041 & 1.0185 & 1.0306 \\
\hline & $C S_{T}(\tau)$ & $1.3405^{*}$ & $1.4009^{*}$ & $1.4586^{* *}$ & 1.1296 & 0.5869 & $1.1693^{*}$ & $2.5175^{* *}$ & $4.8285^{* *}$ & $6.8210^{* *}$ \\
\hline \multirow[t]{2}{*}{$4-26$} & $\hat{\beta}^{+}(\tau)$ & 0.8977 & 0.9112 & 0.9283 & 0.9578 & 0.9699 & 0.9783 & 0.9972 & 1.0198 & 1.0446 \\
\hline & $C S_{T}(\tau)$ & $1.4817^{* *}$ & $1.4740^{* *}$ & $1.4375^{* *}$ & $1.5938^{* *}$ & $1.2855^{*}$ & 0.9978 & $1.1742^{*}$ & $1.1906^{*}$ & $1.6425^{* *}$ \\
\hline \multirow[t]{2}{*}{$13-4$} & $\hat{\beta}^{+}(\tau)$ & 0.9702 & 0.9798 & 0.9949 & 1.0044 & 1.0161 & 1.0258 & 1.0314 & 1.0433 & 1.0702 \\
\hline & $C S_{T}(\tau)$ & $8.5412^{* *}$ & $4.9614^{* *}$ & $3.3197^{* *}$ & $1.3636^{*}$ & 0.6999 & 1.0054 & $1.3845^{*}$ & $1.2737^{*}$ & 1.1013 \\
\hline \multirow[t]{2}{*}{$13-26$} & $\hat{\beta}^{+}(\tau)$ & 0.9725 & 0.9673 & 0.9662 & 0.9780 & 0.9891 & 0.9928 & 1.0019 & 1.0081 & 1.0124 \\
\hline & $C S_{T}(\tau)$ & $2.0737^{* *}$ & $1.5570^{* *}$ & $1.3513^{*}$ & $1.5347^{* *}$ & 1.0337 & 0.9155 & $1.9900^{* *}$ & $3.0591^{* *}$ & $4.8101^{* *}$ \\
\hline \multirow[t]{2}{*}{$26-4$} & $\hat{\beta}^{+}(\tau)$ & 0.9564 & 0.9726 & 0.9982 & 1.0113 & 1.0242 & 1.0339 & 1.0543 & 1.0618 & 1.0496 \\
\hline & $C S_{T}(\tau)$ & $3.7237^{* *}$ & $1.8984^{* *}$ & $1.2797^{*}$ & 0.9947 & 1.1424 & $1.4907^{* *}$ & $1.3302^{*}$ & $1.3294^{*}$ & 1.0870 \\
\hline \multirow[t]{2}{*}{$26-13$} & $\hat{\beta}^{+}(\tau)$ & 0.9878 & 0.9921 & 0.9963 & 1.0029 & 1.0088 & 1.0145 & 1.0288 & 1.0234 & 1.0129 \\
\hline & $C S_{T}(\tau)$ & $4.8220^{* *}$ & $2.9392^{* *}$ & $1.8578^{* *}$ & 0.8833 & 0.9543 & $1.4324^{* *}$ & 1.1671 & $1.3898^{*}$ & $1.8279^{* *}$ \\
\hline \multicolumn{11}{|c|}{ Coupon equivalent } \\
\hline \multirow[t]{2}{*}{$4-13$} & $\hat{\beta}^{+}(\tau)$ & 0.9210 & 0.9445 & 0.9587 & 0.9665 & 0.9748 & 0.9851 & 0.9998 & 1.0101 & 1.0238 \\
\hline & $C S_{T}(\tau)$ & $1.3901^{*}$ & $1.4046^{*}$ & $1.2724^{*}$ & 0.9406 & 0.7558 & 1.0460 & $3.4397^{* *}$ & $4.3309^{* *}$ & $6.8739^{* *}$ \\
\hline \multirow[t]{2}{*}{$4-26$} & $\hat{\beta}^{+}(\tau)$ & 0.8845 & 0.8960 & 0.9117 & 0.9390 & 0.9503 & 0.9604 & 0.9752 & 1.0020 & 1.0222 \\
\hline & $C S_{T}(\tau)$ & $1.4312^{* *}$ & $1.5441^{* *}$ & $1.4277^{* *}$ & $1.3336^{*}$ & 1.1337 & $1.2139^{*}$ & $1.2262^{*}$ & $1.2755^{*}$ & $2.1178^{* *}$ \\
\hline \multirow[t]{2}{*}{$13-4$} & $\hat{\beta}^{+}(\tau)$ & 0.9777 & 0.9861 & 1.0015 & 1.0133 & 1.0231 & 1.0343 & 1.0406 & 1.0512 & 1.0768 \\
\hline & $C S_{T}(\tau)$ & $8.6429^{* *}$ & $5.1868^{* *}$ & $3.4836^{* *}$ & $1.3518^{*}$ & 0.8191 & 1.0880 & $1.2547^{*}$ & $1.3962^{*}$ & 1.1125 \\
\hline \multirow[t]{2}{*}{$13-26$} & $\hat{\beta}^{+}(\tau)$ & 0.9609 & 0.9555 & 0.9553 & 0.9673 & 0.9755 & 0.9798 & 0.9880 & 0.9927 & 0.9979 \\
\hline & $C S_{T}(\tau)$ & $2.3203^{* *}$ & $1.5408^{* *}$ & $1.2494^{*}$ & $1.4563^{* *}$ & 1.0802 & 0.8867 & $1.7646^{* *}$ & $3.1131^{* *}$ & $4.6295^{* *}$ \\
\hline \multirow[t]{2}{*}{$26-4$} & $\hat{\beta}^{+}(\tau)$ & 0.9758 & 0.9926 & 1.0156 & 1.0320 & 1.0473 & 1.0552 & 1.0755 & 1.0807 & 1.0732 \\
\hline & $C S_{T}(\tau)$ & $3.8391^{* *}$ & $2.2514^{* *}$ & $1.5080^{* *}$ & $1.3153^{*}$ & $1.3036^{*}$ & $1.3447^{*}$ & $1.3707^{*}$ & $1.3316^{*}$ & 0.9762 \\
\hline \multirow[t]{2}{*}{$26-13$} & $\hat{\beta}^{+}(\tau)$ & 1.0004 & 1.0045 & 1.0087 & 1.0165 & 1.0218 & 1.0286 & 1.0412 & 1.0360 & 1.0269 \\
\hline & $C S_{T}(\tau)$ & $4.1117^{* *}$ & $2.9041^{* *}$ & $1.7448^{* *}$ & 0.8042 & 1.0676 & $1.3622^{*}$ & $1.2202^{*}$ & $1.3708^{*}$ & $1.8768^{* *}$ \\
\hline
\end{tabular}

US daily Treasury bill data is from $01 / 02 / 2002$ to $04 / 30 / 2012$. The number of observations is 2585 . Let * denote rejection of the null of cointegration at $5 \%$ level and let ${ }^{* *}$ denote rejection at $1 \%$ level. 
Table 2: Cointegration among financial commercial paper rates (yields: 30, 60, 90 days).

\begin{tabular}{llrrrrrrrrr}
\hline $\boldsymbol{y}-\boldsymbol{x}$ & & $\boldsymbol{\tau}=\mathbf{0 . 1}$ & $\mathbf{0 . 2}$ & $\mathbf{0 . 3}$ & $\mathbf{0 . 4}$ & $\mathbf{0 . 5}$ & $\mathbf{0 . 6}$ & $\mathbf{0 . 7}$ & $\mathbf{0 . 8}$ & $\mathbf{0 . 9}$ \\
\hline $30-60$ & $\hat{\beta}^{+}(\tau)$ & 1.0056 & 1.0036 & 1.0026 & 1.0039 & 1.0041 & 1.0041 & 1.0041 & 1.0052 & 1.0156 \\
& $C S_{T}(\tau)$ & $1.5898^{* *}$ & $2.4501^{* *}$ & $2.0793^{* *}$ & 1.1205 & 1.1426 & $1.7477^{* *}$ & $3.7407^{* *}$ & $6.6798^{* *}$ & $4.6846^{* *}$ \\
$30-90$ & $\hat{\beta}^{+}(\tau)$ & 1.0069 & 1.0062 & 1.0047 & 1.0061 & 1.0082 & 1.0096 & 1.0090 & 1.0101 & 1.0326 \\
& $C S_{T}(\tau)$ & $1.5954^{* *}$ & $2.3190^{* *}$ & $2.5919^{* *}$ & $2.3279^{* *}$ & $1.7541^{* *}$ & $1.8461^{* *}$ & $2.5133^{* *}$ & $4.4216^{* *}$ & $2.9653^{* *}$ \\
$60-30$ & $\hat{\beta}^{+}(\tau)$ & 0.9828 & 0.9947 & 0.9961 & 0.9962 & 0.9962 & 0.9963 & 0.9964 & 0.9950 & 0.9926 \\
& $C S_{T}(\tau)$ & $6.0914^{* *}$ & $6.9691^{* *}$ & $4.4223^{* *}$ & $2.2500^{* *}$ & $1.2470^{* *}$ & 1.1233 & $1.6134^{* *}$ & $2.0012^{* *}$ & $1.7048^{* *}$ \\
$60-90$ & $\hat{\beta}^{+}(\tau)$ & 1.0095 & 1.0063 & 1.0043 & 1.0041 & 1.0043 & 1.0042 & 1.0047 & 1.0049 & 1.0102 \\
& $C S_{T}(\tau)$ & $1.4585^{* *}$ & $2.3730^{* *}$ & $1.7650^{* *}$ & 1.0464 & 0.9105 & $1.5000^{* *}$ & $3.8005^{* *}$ & $6.4077^{* *}$ & $6.6321^{* *}$ \\
$90-30$ & $\hat{\beta}^{+}(\tau)$ & 0.9656 & 0.9885 & 0.9893 & 0.9903 & 0.9922 & 0.9936 & 0.9928 & 0.9906 & 0.9898 \\
& $C S_{T}(\tau)$ & $3.3449^{* *}$ & $4.2920^{* *}$ & $2.7547^{* *}$ & $1.9796^{* *}$ & $1.4799^{* *}$ & $2.1144^{* *}$ & $2.3797^{* *}$ & $2.1914^{* *}$ & $1.6938^{* *}$ \\
$90-60$ & $\hat{\beta}^{+}(\tau)$ & 0.9877 & 0.9950 & 0.9954 & 0.9960 & 0.9960 & 0.9943 & 0.9940 & 0.9938 & 0.9896 \\
& $C S_{T}(\tau)$ & $7.7150^{* *}$ & $7.0606^{* *}$ & $4.1914^{* *}$ & $1.8809^{* *}$ & 0.8963 & $1.5375^{* *}$ & $1.4186^{* *}$ & $2.0230^{* *}$ & $1.9401^{* *}$ \\
\hline
\end{tabular}

US daily financial commercial paper rate data is from $01 / 02 / 1997$ to $04 / 30 / 2012$. The number of observations is 3748 .

Table 3: Cointegration among Treasury constant maturity rates (yields: 3, 6, 12 months).

\begin{tabular}{llrrrrrrrrr}
\hline $\boldsymbol{y}-\boldsymbol{x}$ & & $\boldsymbol{\tau}=\mathbf{0 . 1}$ & $\mathbf{0 . 2}$ & $\mathbf{0 . 3}$ & $\mathbf{0 . 4}$ & $\mathbf{0 . 5}$ & $\mathbf{0 . 6}$ & $\mathbf{0 . 7}$ & $\mathbf{0 . 8}$ & $\mathbf{0 . 9}$ \\
\hline $3-6$ & $\hat{\beta}^{+}(\tau)$ & 0.9465 & 0.9605 & 0.9660 & 0.9687 & 0.9730 & 0.9780 & 0.9828 & 0.9880 & 0.9924 \\
& $C S_{T}(\tau)$ & 0.8859 & 1.1161 & $1.3232^{*}$ & $1.2241^{*}$ & $1.2130^{*}$ & $1.2300^{*}$ & $1.1889^{*}$ & $1.3899^{*}$ & $1.4494^{* * *}$ \\
$3-12$ & $\hat{\beta}^{+}(\tau)$ & 0.9071 & 0.9338 & 0.9462 & 0.9499 & 0.9560 & 0.9642 & 0.9742 & 0.9879 & 1.0071 \\
& $C S_{T}(\tau)$ & 0.8836 & $1.2439^{*}$ & $1.4801^{* *}$ & $1.4236^{*}$ & $1.3526^{*}$ & $1.4633^{* *}$ & $1.6802^{* *}$ & $1.7879^{* * *}$ & $1.7508^{* *}$ \\
$6-3$ & $\hat{\beta}^{+}(\tau)$ & 1.0056 & 1.0104 & 1.0161 & 1.0210 & 1.0265 & 1.0305 & 1.0334 & 1.0373 & 1.0517 \\
& $C S_{T}(\tau)$ & $1.9344^{* *}$ & $1.6523^{* *}$ & $1.3564^{*}$ & $1.2062^{*}$ & $1.2235^{*}$ & $1.2491^{*}$ & $1.2896^{*}$ & 1.1003 & 1.0039 \\
$6-12$ & $\hat{\beta}^{+}(\tau)$ & 0.9594 & 0.9730 & 0.9772 & 0.9807 & 0.9845 & 0.9899 & 0.9956 & 1.0028 & 1.0133 \\
& $C S_{T}(\tau)$ & 1.0650 & 1.0816 & $1.3913^{*}$ & $1.6695^{* *}$ & $1.6807^{* *}$ & $1.9304^{* *}$ & $2.0034^{* *}$ & $2.0739^{* *}$ & $2.0344^{* *}$ \\
$12-3$ & $\hat{\beta}^{+}(\tau)$ & 0.9846 & 1.0064 & 1.0211 & 1.0293 & 1.0363 & 1.0432 & 1.0507 & 1.0605 & 1.0839 \\
& $C S_{T}(\tau)$ & $1.9336^{* *}$ & $1.8389^{* *}$ & $1.6954^{* *}$ & $1.7005^{* *}$ & $1.5964^{* *}$ & $1.6644^{* *}$ & $1.7136^{* *}$ & $1.6424^{* *}$ & $1.2180^{*}$ \\
$12-6$ & $\hat{\beta}^{+}(\tau)$ & 0.9808 & 0.9952 & 1.0037 & 1.0079 & 1.0111 & 1.0173 & 1.0209 & 1.0253 & 1.0377 \\
& $C S_{T}(\tau)$ & $2.2030^{* *}$ & $2.1713^{* *}$ & $2.0231^{* *}$ & $2.0425^{* *}$ & $2.0922^{* *}$ & $1.7259^{* *}$ & $1.5582^{* *}$ & $1.2095^{*}$ & 0.9516 \\
\hline
\end{tabular}

US daily Treasury bill constant maturity data is from 01/04/1982 to 04/30/2012. The number of observations is 7584 .

From the second panel of Table 1, the corresponding coupon equivalent Treasury bill rates follow a similar pattern. There is evidence of cointegration when the quantile level is between 0.4 and 0.6. The upper quantiles of the 13- and 26-week coupon equivalent rates are cointegrated with the 4-week rate.

In Table 2, for the 30-, 60-, and 90-day financial commercial rates, the null of cointegration is retained around the median in most cases. However, the distributions of the 30- and 90-day rates are not cointegrated in either direction. In addition, the fully modified coefficient estimates $\hat{\beta}^{+}(\tau)$ are highly significant in the cases when cointegration is not rejected.

The results from the model with the Treasury constant maturities interest rates or the Treasury yield curve rates are different. In many cases, the null of cointegration is retained in either the lower or upper tail. In Table 3, the Treasury constant maturities interest rates of shorter terms, such as the 3- and 6-month rates, are cointegrated with rates of longer terms in the lower tail of the distribution. The 6-month constant maturities rate is cointegrated with the 3-month rate in the upper tail. Similarly, there is cointegration relationship between the $90 \%$ quantile of the 12-month rate and the 6-month rate. However, the distribution of the 12-month rate is not cointegrated with the 3 -month rate. Also, when cointegration is not rejected, the fully modified coefficient estimates $\hat{\beta}^{+}(\tau)$ are highly significant.

In general, the expectations hypothesis for the term structure is supported in the inner quantiles for Treasury bill rates and financial commercial rates. For the constant maturity Treasury rates, cointegration 
relationships are found in the tails of the interest rate distributions. One explanation may be that each interest rate itself exhibits asymmetric adjustment dynamics over the business cycle ${ }^{9}$ (Koenker and Xiao 2004). Hence, results from the multifactor (term structure) model also display some asymmetry in the median, lower, or upper quartiles of the interest rate distributions.

\section{Conclusion}

This paper provides a cumulated sum test for the null hypothesis of quantile cointegration. In order to correct serial correlation and long run endogeneity, a Phillips-Hansen type fully modified quantile estimator for the cointegrating coefficients is employed to remove the second-order bias and nuisance parameters. For this semiparametric correction, the long run covariance between the regression disturbance and the innovation of the I(1) regressors is estimated using the Bartlett kernel with the plug-in bandwidth, as suggested by Andrews (1991) and Xiao and Phillips (2002). The CUSUM test statistic is composed of the partial sums of the residuals from the fully modified quantile regression. For each quantile, under the null of cointegration, the test statistic converges to a functional of Brownian motions.

The model is applied to several sets of daily US interest rate data. The US interest rates are found to be nonstationary and integrated of order one. Over various quantiles, evidence of cointegration is mixed. In the conditional quantile context, the expectations hypothesis for the term structure is retained only in part of the interest rate distribution for certain data sets.

In addition, several extensions can be made to the model. For example, it is relevant to incorporate structural changes in the quantile regression, since the long run relationship among the nonstationary variables may not be time-invariant. Also, ignoring the possibility of structural break can affect the power of cointegration tests (Gregory 1994; Gregory and Hansen 1996). In empirical studies, for example, Hansen (1992), Hall, Anderson, and Granger (1992), and Mandeno and Giles (1995) all consider a possible regime shift in the term structure data due to the Federal Reserve's policy change. By allowing for structural changes, one can estimate and test the quantile cointegration relationship, which is possibly unstable over time.

Funding: Supported by the Fundamental Research Funds for the Central Universities, and the Research Funds of Renmin University of China 14XNF018.

\section{Appendix}

\section{A Proofs}

\section{A.1 Proof of Theorem 1}

Theorem 1 can be proved following the procedure in Section A.1 from Xiao (2009), since under the null hypothesis of cointegration the model in this paper has the same asymptotic behavior as the quantile cointegrating regression considered by Xiao (2009). Let " $\Sigma$ " denote summation over $t$ from $t=1$ to $t=T$ unless otherwise specified. For a single quantile $\tau$, the unknown quantile regression coefficients $\hat{\theta}(\tau)$ are estimated by the minimization problem:

$$
\hat{\theta}(\tau)=\left(\hat{\alpha}^{\prime}(\tau), \hat{\beta}^{\prime}(\tau)\right)^{\prime}=\arg \min _{\theta} \sum \rho_{\tau}\left(y_{t}-z_{t}^{\prime} \theta\right) .
$$

9 Koenker and Xiao (2004) apply quantile autoregression based unit root tests to several interest rate series in the US. They find that short-term interest rate series are not constant unit root processes over different quantiles. There is significant support for asymmetry in the business cycle dynamics of the interest rates. 
If $\hat{\theta}(\tau)$ is a minimizer of the above objective function, then $\hat{\phi}$ is the solution to the following minimization problem:

$$
\min _{\phi} \sum\left\{\rho_{\tau}\left(u_{t}(\tau)-\left(D_{T}^{-1} \phi\right)^{\prime} z_{t}\right)-\rho_{\tau}\left(u_{t}(\tau)\right)\right\}
$$

where $\hat{\phi}=D_{T}(\hat{\theta}(\tau)-\theta(\tau))$. Consider the objective function

$$
G_{T}(\phi)=\sum\left\{\rho_{\tau}\left(u_{t}(\tau)-\left(D_{T}^{-1} \phi\right)^{\prime} z_{t}\right)-\rho_{\tau}\left(u_{t}(\tau)\right)\right\}
$$

and $\hat{\phi}$ is a minimizer of $G_{T}(\phi)$. Since the check function $\rho_{\tau}(\cdot)$ is convex, the objective function $G_{T}(\phi)$ is also convex, which is similar to the convex random function discussed in Knight (1989) ${ }^{10}$. According to Lemma A of Knight (1989), if the finite-dimensional distributions of $G_{T}(\phi)$ converge weakly to those of $G(\phi)$ and $G(\phi)$ has a unique minimum, the convexity of $G_{T}(\phi)$ implies that $\hat{\phi}$ converges in distribution to the minimizer of $G(\phi) .{ }^{11}$ It will be shown later that $G(\phi)$ is convex and differentiable so that it has a unique minimizer.

To derive the asymptotic distribution of the consistent estimator $\hat{\theta}(\tau)$, we have

$$
\begin{aligned}
G_{T}(\phi)= & -\sum\left(D_{T}^{-1} \phi\right)^{\prime} z_{t} \psi_{\tau}\left(u_{t}(\tau)\right)+\sum\left(u_{t}(\tau)-\left(D_{T}^{-1} \phi\right)^{\prime} z_{t}\right) \\
& \times\left[I\left(0>u_{t}(\tau)>\left(D_{T}^{-1} \phi\right)^{\prime} z_{t}\right)-I\left(0<u_{t}(\tau)<\left(D_{T}^{-1} \phi\right)^{\prime} z_{t}\right)\right] .
\end{aligned}
$$

From the invariance principle stated in Assumption 1 and Assumption 2, for the first term,

$$
\begin{aligned}
\phi^{\prime} D_{T}^{-1} \sum z_{t} \psi_{\tau}\left(u_{t}(\tau)\right) & \Rightarrow \phi^{\prime}\left[\begin{array}{c}
\int B_{d} d B_{\psi} \\
\int B_{x} d B_{\psi}+\Delta_{x \psi}
\end{array}\right] \\
& =\phi^{\prime}\left[\int B_{z} d B_{\psi}+\bar{\Delta}_{x \psi}\right],
\end{aligned}
$$

where $B_{z}=\left(B_{d}^{\prime}, B_{x}^{\prime}\right)^{\prime}, \bar{\Delta}_{v \psi}=\left(0, \Delta_{x \psi}^{\prime}\right)^{\prime}$, and $\Delta_{x \psi}=\sum_{t=0}^{\infty} E\left(\xi_{2 t} \psi_{\tau}\left(u_{0}(\tau)\right)\right)$ is the one-sided long run covariance between $\xi_{2 t}$ and $\psi_{\tau}\left(u_{t}(\tau)\right)$.

Similar to the derivation on page 257-258 in Appendix A.1 from Xiao (2009), under Assumption 3 the second term converges to

$$
\frac{1}{2} f\left(F^{-1}(\tau)\right) \phi^{\prime}\left[\int B_{z} B_{z}^{\prime}\right] \phi
$$

Consequently,

$$
\begin{aligned}
G_{T}(\phi) & \Rightarrow-\phi^{\prime}\left[\int B_{z} d B_{\psi}+\bar{\Delta}_{x \psi}\right]+\frac{1}{2} f\left(F^{-1}(\tau)\right) \phi^{\prime}\left[\int B_{z} B_{z}^{\prime}\right] \phi \\
& :=G(\phi),
\end{aligned}
$$

where ":=" signifies definitional equality. Since $G(\phi)$ is convex and differentiable, there is only one solution to the minimization problem of $G(\phi)$. From the first order condition that

$$
\frac{\partial G(\phi)}{\partial \phi^{\prime}}=-\left[\int B_{z} d B_{\psi}+\bar{\Delta}_{x \psi}\right]+\frac{1}{2} f\left(F^{-1}(\tau)\right)\left[\int B_{z} B_{z}^{\prime}\right] \phi=0,
$$

the unique minimizer of $G(\phi)$ is

$$
\left[f\left(F^{-1}(\tau)\right) \int B_{z} B_{z}^{\prime}\right]^{-1}\left[\int B_{z} d B_{\psi}+\bar{\Delta}_{x \psi}\right]
$$

10 Refer to the discussion of equation (1) on page 262 in Knight (1989). The objective function here is essentially the same as equation (1) of Knight (1989).

11 This follows directly from Lemma A in the appendix on page 276 of Knight (1989). Both Knight (1989) and Pollard (1991) consider the convexity property when deriving the asymptotic distribution of the least-absolute-deviations (LAD) estimator defined by minimizing the sum of the absolute values of the residuals. Note that the LAD estimator is a special case of the quantile regression estimator when $\tau=0.5$. 
By Lemma A of Knight (1989) and the convexity lemma of Pollard (1991), ${ }^{12}$ since $G_{T}(\phi)$ is minimized at $\hat{\phi}=D_{T}(\hat{\theta}(\tau)-\theta(\tau))$, we obtain the limit distribution of the regression coefficient estimator such that

$$
D_{T}(\hat{\theta}(\tau)-\theta(\tau)) \Rightarrow\left[f\left(F^{-1}(\tau)\right) \int B_{z} B_{z}^{\prime}\right]^{-1}\left[\int B_{z} d B_{\psi}+\bar{\Delta}_{x \psi}\right] .
$$

\section{A.2 Proof of Theorem 2}

Similar to the case of fully modified OLS regression, the fully modified quantile estimator is consistent, which is also shown in the following derivation. The regression coefficient estimator after the modification $\hat{\theta}^{+}(\tau)=\left(\hat{\alpha}^{\prime}(\tau), \hat{\beta}^{\prime+}(\tau)\right)^{\prime}$ is

$$
\hat{\theta}^{+}(\tau)=\hat{\theta}(\tau)-\left[\overline{f\left(F^{-1}(\tau)\right)} \sum z_{t} z_{t}^{\prime}\right]^{-1}\left[\sum z_{t} \hat{\Omega}_{\psi x} \hat{\Omega}_{x x}^{-1} \Delta x_{t}+T \overline{\hat{\Delta}}_{x \psi}^{+}\right],
$$

where $\overline{\hat{\Delta}}_{x \psi}^{+}=\left(0, \Delta_{x \psi}^{++}\right)^{\prime}$. Under cointegration and Assumptions 1-3, Theorem 1 holds so that

$$
\begin{aligned}
& D_{T}(\hat{\theta}(\tau)-\theta(\tau)) \\
& \Rightarrow\left[f\left(F^{-1}(\tau)\right) \int B_{z} B_{z}^{\prime}\right]^{-1}\left[\int B_{z} d B_{\psi \cdot x}+\int B_{z} d \Omega_{\psi x} \Omega_{x x}^{-1} B_{x}+\bar{\Delta}_{x \psi}\right] .
\end{aligned}
$$

Then, we have

$$
\begin{aligned}
& D_{T}\left(\hat{\theta}^{+}(\tau)-\theta(\tau)\right)=D_{T}(\hat{\theta}(\tau)-\theta(\tau)) \\
& -D_{T}\left[\overline{f\left(F^{-1}(\tau)\right)} \sum z_{t} z_{t}^{]^{-1}}\right]^{-1}\left[z_{t} \hat{\Omega}_{\psi x} \hat{\Omega}_{x x}^{-1} \Delta x_{t}+T \overline{\hat{\Delta}}_{x \psi}^{+}\right] \\
& \Rightarrow\left[f\left(F^{-1}(\tau)\right) \int B_{z} B_{z}^{\prime}\right]^{-1} \int B_{z} d B_{\psi \cdot x} \\
& \sim M N\left(0, \frac{\omega_{\psi \cdot x}^{2}}{f\left(F^{-1}(\tau)\right)^{2}}\left[\int B_{z} B_{z}^{\prime}\right]^{-1}\right),
\end{aligned}
$$

where $\omega_{\psi \cdot x}^{2}=\omega_{\psi}^{2}-\Omega_{\psi x} \Omega_{x x}^{-1} \Omega_{x \psi}$. Similar to $\hat{\theta}(\tau)$, the fully modified estimator $\hat{\theta}^{+}(\tau)$ is also consistent, with $D_{T}\left(\hat{\theta}^{+}(\tau)-\theta(\tau)\right)=O_{p}(1)$.

\section{A.3 Proof of Theorem 3}

For the fully modified quantile regression, we have $y_{t}^{+}=y_{t}-\hat{\Omega}_{\psi x} \hat{\Omega}_{x x}^{-1} \Delta x_{t}$ and $\hat{u}_{t}^{+}(\tau)=y_{t}^{+}-z_{t}^{\prime} \hat{\theta}^{+}(\tau)$. The residuals of the quantile regression are transformed to $\psi_{\tau}\left(\hat{u}_{t}^{+}(\tau)\right)=\tau-I\left(\hat{u}_{t}^{+}(\tau)<0\right)$. Under cointegration and Assumptions 1-3, the partial sum of $\psi_{\tau}\left(\hat{u}_{t}^{+}(\tau)\right)$ is

$$
\begin{aligned}
T^{-\frac{1}{2}} \sum_{t=1}^{[T r]} \psi_{\tau}\left(\hat{u}_{t}^{+}(\tau)\right) & \Rightarrow B_{\psi \cdot x}-\left[\int d B_{\psi \cdot x} B_{z}^{\prime}\right]\left[\int B_{z} B_{z}^{\prime}\right]^{-1} \int_{0}^{r} B_{z}:=\underline{B}_{\psi \cdot x} \\
& =\omega_{\psi \cdot x}\left\{W_{1}-\left[\int d W_{1} S^{\prime}\right]\left[\int S S^{\prime}\right]^{-1} \int_{0}^{r} S\right\}:=\omega_{\psi \cdot x} \underline{W},
\end{aligned}
$$

where in general $S=\left(B_{d}^{\prime}, W_{2}^{\prime}\right)^{\prime}$ and $W_{1}$ and $W_{2}$ are one- and $k$-dimensional standard Brownian motions that are independent of each other. If the regression contains an intercept but no time trend, then $S=\left(1, W_{2}^{\prime}\right)^{\prime}$. Also, by definition $\underline{W}=W_{1}-\left[\int d W_{1} S^{\prime}\right]\left[\int S S^{\prime}\right]^{-1} \int_{0}^{r} S$.

12 Similar to the argument of Knight (1989), Pollard (1991) approximates the objective function as $G_{T}(\phi)$ by a quadratic function as $G(\phi)$ and shows that $\hat{\phi}$ lies close enough to the minimizing value of $G(\phi)$ to share its asymptotic behavior. 
The CUSUM test statistic is given by

$$
\begin{aligned}
C S_{T}(\tau) & =\max _{n=1, \ldots, T} \frac{1}{\hat{\omega}_{\psi \cdot x} \sqrt{T}}\left|\sum_{t=1}^{n} \psi_{\tau}\left(\hat{u}_{t}^{+}(\tau)\right)\right| \\
& \Rightarrow \sup _{0 \leq r \leq 1}\left|\left\{W_{1}-\left[\int d W_{1} S^{\prime}\right]\left[\int S S^{\prime}\right]^{-1} \int_{0}^{r} S\right\}\right|,
\end{aligned}
$$

where $\hat{\omega}_{\psi \cdot x}^{2}=\hat{\omega}_{\psi}^{2}-\hat{\Omega}_{\psi x} \hat{\Omega}_{x x}^{-1} \hat{\Omega}_{x \psi}$.

\section{A.4 Proof of Theorem 4}

Under $H_{1}$ we have

$$
\hat{\beta}(\tau) \Rightarrow\left[f\left(F^{-1}(\tau)\right) \int \underline{B}_{x d} \underline{B}_{x d}^{\prime}\right]^{-1}\left(\int \underline{B}_{x d} \underline{B}_{y d}\right)=\varsigma_{d}(\tau),
$$

where $\underline{B}_{y d}=B_{y}-\left(\int B_{y} d^{\prime}\right)\left(\int d B_{d}^{\prime}\right)^{-1} B_{d}$. Then

$$
\begin{aligned}
& T^{-\frac{1}{2}} \psi_{\tau}\left(\hat{u}_{[T r]}(\tau)\right) \Rightarrow \underline{B}_{y d}-\left(\int \underline{B}_{y d} \underline{B}_{x d}^{\prime}\right)\left[f\left(F^{-1}(\tau)\right) \int \underline{B}_{x d} \underline{B}_{x d}^{\prime}\right]^{-1} \underline{B}_{x d}:=\underline{P} \\
& T^{-\frac{3}{2}} \sum_{t=1}^{[T r]} \psi_{\tau}\left(\hat{u}_{[T r]}(\tau)\right) \Rightarrow \int_{0}^{r} \underline{P}:=\bar{P} .
\end{aligned}
$$

Analogues to Lemma 1 from Xiao and Phillips (2002), under $H_{1}$ as $T \rightarrow \infty, M \rightarrow \infty$, and $M / T \rightarrow 0$,

$$
\begin{aligned}
\frac{1}{M} \hat{\Omega}_{x \psi} & \Rightarrow 2 \pi \tilde{K}(0)\left(\int d B_{x} \underline{P}\right)+\Omega_{*}, \\
\frac{1}{M} \hat{\Delta}_{x \psi} & \Rightarrow 2 \pi \tilde{K}_{1}(0)\left(\int d B_{x} \underline{P}\right)+\Delta_{*}, \\
\frac{1}{T M} \hat{\omega}_{\psi \cdot x}^{2} & \Rightarrow 2 \pi \tilde{K}(0) \int \underline{P}^{2},
\end{aligned}
$$

where $\quad \Omega_{*}=\Omega_{x w} \eta, \quad \Delta_{*}=\Delta_{x w} \eta, \quad \eta=\left(1,-\varsigma_{d}^{\prime}(\tau)\right)^{\prime}, \quad \Omega_{x w}=\left[\Omega_{x \psi}: \Omega_{x x}\right], \quad \Delta_{x w}=\left[\Delta_{x \psi}: \Delta_{x x}\right], \quad \tilde{K}(0)=\frac{1}{2 \pi} \int K(x) d x, \quad$ and $\tilde{K}_{1}(0)=\lim _{M \rightarrow \infty} \frac{1}{2 \pi M} \sum_{h=0}^{M} K\left(\frac{h}{M}\right)$. With the modified residuals,

$$
\frac{1}{T}\left[\max _{n=1, \ldots, T} \frac{n}{\sqrt{T}}\left|\frac{1}{n} \sum_{t=1}^{n} \psi_{\tau}\left(\hat{u}_{t}^{+}(\tau)\right)\right|\right] \Rightarrow \sup _{0 \leq r \leq 1}|\underline{P}(r)| .
$$

Since $\hat{\omega}_{\psi \cdot x}=O_{p}\left(T^{\frac{1}{2}} M^{\frac{1}{2}}\right)$, under $H_{1}$ we have $C S_{T}(\tau)=O_{p}\left(T^{\frac{1}{2}} M^{-\frac{1}{2}}\right) \rightarrow \infty$.

\section{References}

Andrews, D. W. K. 1991. “Heteroskedasticity and Autocorrelation Consistent Covariance Matrix Estimation.” Econometrica 59 (3): 817-858.

Boothe, P. 1991. “Interest Parity, Cointegration, and the Term Structure in Canada and the United States." The Canadian Journal of Economics 24 (3): 595-603.

Brown, R. L., and J. Durbin. 1968. Methods of Investigating Whether a Regression Relationship Is Constant over Time. Selected Statistical Papers, European Meeting, Mathematical Centre Tracts No. 26, Amsterdam.

Brown, R. L., J. Durbin, and J. Evans. 1975. "Techniques for Testing the Constancy of Regression Relationship over Time." Journal of Royal Statistical Society, Series B, 37: 149-163.

Campbell, J. Y. 1987. “Does Saving Anticipate Declining Labor Income? An Alternative Test of the Permanent Income Hypothesis.” Econometrica 55 (6): 1249-1273. 
Campbell, J. Y., and R. J. Shiller. 1987. “Cointegration and Tests of Present Value Models.” Journal of Political Economy 95 (5): 1062-1088.

Campbell, J. Y., and R. J. Shiller. 1988a. "Stock Prices, Earnings and Expected Dividends." The Journal of Finance 43 (3): 661-676.

Campbell, J. Y., and R. J. Shiller. 1988b. "The Dividend-Price Ratio and Expectations of Future Dividends and Discount Factors." Review of Financial Studies 1 (3): 195-228.

Downing, C., and S. Oliner. 2007. "The Term Structure of Commercial Paper Rate." Journal of Financial Economics 83 (1): 59-86.

Engle, R. F., and C. W. Granger. 1987. "Co-integration and Error Correction: Representation, Estimation, and Testing." Econometrica 55 (2): 251-276.

Gregory, A. W. 1994. "Testing for Cointegration in Linear Quadratic Models.” Journal of Business and Economic Statistics 12 (3): 347-360.

Gregory, A. W., and B. E. Hansen. 1996. "Residual-Based Tests for Cointegration in Models with Regime Shifts." Journal of Econometrics 70 (1): 99-126.

Hall, A. D., H. M. Anderson, and C. W. J. Granger. 1992. "A Cointegration Analysis of Treasury Bill Yields.” The Review of Economics and Statistics 74 (1): 116-126.

Hansen, B. E. 1992. "Tests for Parameter Instability in Regressions with I(1) Processes." Journal of Business and Economic Statistics 10 (3): 321-335.

Hao, K., and B. Inder. 1996. "Diagnostic Test for Structural Change in Cointegrated Regression Models." Economics Letters 50 (2): 179-187.

lacone, F. 2009. “A Semiparametric Analysis of the Term Structure of the U.S. Interest Rates." Oxford Bulletin of Economics and Statistics 71 (4): 475-490.

Johansen, S. 1988. "Statistical Analysis of Cointegrating Vectors." Journal of Economic Dynamics and Control 12: 231-254.

Johansen, S. 1991. "Estimation and Hypothesis Testing of Cointegration Vectors in Gaussian Vector Autoregressive Models." Econometrica 59 (6): 1551-1580.

Johansen, S. 1995. Likelihood-Based Inference in Cointegrated Vector Autoregressive Models. Oxford: Oxford University Press. Knight, K. 1989. "Limit Theory for Autoregressive-Parameter Estimates in an Infinite-Variance Random Walk." The Canadian Journal of Statistics 17 (3): 261-278.

Koenker, R., and G. Bassett. 1978. “Regression Quantiles.” Econometrica 46 (1): 33-49.

Koenker, R., and F. K. Hallock. 2001. "Quantile Regression.” Journal of Economic Perspectives 15 (4): 143-156.

Koenker, R., and Z. Xiao. 2004. "Unit Root Quantile Autoregression Inference." Journal of the American Statistical Association 99 (467): 775-787.

Kuriyama, N. 2015. “Inference in Quantile Cointegrating Regressions with Structural Changes.” Working paper.

Mandeno, R. J., and D. E. A. Giles. 1995. "The Expectations Theory of the Term Structure: A Cointegration/Causality Analysis of U.S. Interest Rates.” Applied Financial Economics 5 (5): 273-283.

Oka, T., and Z. Qu. 2011. “Estimating Structural Changes in Regression Quantiles.” Journal of Econometrics 162 (2): 248-267.

Park, J. 1990. "Testing for Unit Roots and Cointegration by Variable Addition." In Advances in Econometrics, edited by T. B. Fomby and G. F. Rhodes, Jr., 107-133. Greenwich, Connecticut: JAI Press.

Park, J., S. Ouliaris, and B. Choi. 1988. "Spurious Regressions and Tests for Cointegration”. CAE Working paper 88-07, Cornell University.

Phillips, P. C. B., and B. E. Hansen. 1990. "Statistical Inference in Instrumental Variables Regression with I(1) Processes.” Review of Economic Studies 57 (1): 99-125.

Phillips, P. C. B., and M. Loretan. 1991. “Estimating Long Run Economic Equilibria.” Review of Economic Studies 58 (3): $407-436$.

Phillips, P. C. B., and S. Ouliaris. 1990. “Asymptotic Properties of Residual-Based Tests for Cointegration.” Econometrica 58 (1): 165-193.

Ploberger, W., and W. Kramer. 1992. “The CUSUM Test with OLS Residuals.” Econometrica 60 (2): 271-285.

Pollard, D. 1991. “Asymptotics for Least Absolute Deviation Regression Estimators.” Econometric Theory 7 (2): $186-199$.

Qu, Z. 2008. "Testing for Structural Change in Regression Quantiles.” Journal of Econometrics 146 (1): 170-184.

Stock, J. H., and M. W. Watson. 1988. "Testing for Common Trends.” Journal of the American Statistical Association 83 (404): 1097-1107.

Shin, Y. 1994. “A Residual Based Test of the Null of Cointegration Against the Alternative of No Cointegration.” Econometric Theory 10 (1): 91-115.

Xiao, Z. 2009. "Quantile Cointegrating Regression.” Journal of Econometrics 150 (2): 248-260.

Xiao, Z., and P. C. B. Phillips. 2002. "A CUSUM Test for Cointegration Using Regression Residuals.” Journal of Econometrics 108 (1): 43-61.

Supplemental Material: The online version of this article (DOI: 10.1515/snde-2013-0107) offers supplementary material, available to authorized users. 